\title{
Erratum to: Spheroid-plug model as a tool to study tumor development, angiogenesis, and heterogeneity in vivo
}

\author{
Krzysztof Szade $^{1,2}$ - Monika Zukowska ${ }^{1}$ - Agata Szade $^{1} \cdot$ Guillaume Collet $^{2,4}$. \\ Damian Kloska $^{1}$ - Claudine Kieda ${ }^{2,3}$ • Alicja Jozkowicz ${ }^{1}$. Jozef Dulak ${ }^{1,3}$
}

Published online: 27 May 2016

(C) International Society of Oncology and BioMarkers (ISOBM) 2016

Erratum to: Tumor Biol. (2016) 37:2481-2496

DOI 10.1007/s13277-015-4065-z

Unfortunately, the original version of this article contained an error in one of the grant number listed in the acknowledgment section.

The corrected grant number in acknowledgment section is shown below in bold.

Acknowledgments This study was supported by the following grants: Ventures (2011-8/9) granted to K.Sz. by Foundation for Polish Science, Harmonia (2012/06/M/NZ1/ 00008) granted to A.J. by Polish National Science Center, and National Centre for Research and Development granted to J.D. (PBS2/B7/15/2014). Research was conducted in the scope of the MiR-TANGo International Associated Laboratory (LIA). The FEDER from the European Union grant no. 5788/39114 supported C.K. The Faculty of Biochemistry, Biophysics and Biotechnology of the Jagiellonian University was beneficiary of the structural funds from the European Union (grant POIG.02.01.00-12064/08). The Faculty of Biochemistry, Biophysics and Biotechnology of Jagiellonian University is a partner of the Leading National Research Center (KNOW) supported by the Ministry of Science and Higher Education. We would like to thank administrative staff Agnieszka AndrychowiczRog and Joanna Uchto as well as animal facility members Janusz Drebot, Karolina Hajduk, Elzbieta Slizewska, and Ewa Werner for their valuable help.

The online version of the original article can be found at http://dx.doi.org/ 10.1007/s13277-015-4065-z.

Alicja Jozkowicz

alicja.jozkowicz@uj.edu.pl

$\triangle$ Jozef Dulak

jozef.dulak@uj.edu.pl

1 Department of Medical Biotechnology, Faculty of Biochemistry, Biophysics and Biotechnology, Jagiellonian University,

Krakow, Poland

2 Centre for Molecular Biophysics, Cell Recognition and Glycobiology, UPR4301-CNRS, Orleans, France

3 Malopolska Centre of Biotechnology, Krakow, Poland

4 Present address: Skaggs School of Pharmacy and Pharmaceutical Sciences, Laboratory of Bioresponsive Materials, University of California, San Diego, CA, USA 